\title{
Um Sistema e-Health Inteligente para Análise e Detecção de Doenças Cardíacas
}

\author{
Diego A. B. Moreira ${ }^{1}$, Levy G. Chaves ${ }^{1}$, Rafael L. Gomes ${ }^{1}$, Joaquim Celestino Jr ${ }^{1}$ \\ ${ }^{1}$ Centro de Ciência e Tecnologia (CCT) - Universidade Estadual do Ceará (UECE) \\ \{diego, levy, rafaellgom, celestino\}@larces.uece.br
}

\begin{abstract}
Resumo. A indústria de cuidados médicos vem evoluindo a sua prestação de serviços, focando na qualidade do atendimento ao paciente. Tem-se então a necessidade de uma infraestrutura para monitoramento da saúde dos pacientes a partir da análise dos dados destes pacientes, com intuito de detectar possíveis doenças, dentre elas, doenças cardíacas. Dentro deste contexto, este artigo apresenta um sistema e-Health inteligente para analisar os dados biologicos dos pacientes e detectar possíveis complicações cardíacas. No sistema proposto aplica-se três fases: (I) trata-se os dados através de filtros; (II) técnicas para extração das informações úteis; e, (III) os dados são analisados e classificados para detectar doenças cardiacas. Os experimentos realizados utilizando-se uma base dados real mostram que o sistema proposto é capaz de classificar com alta acurácia as anomalias cardíacas.
\end{abstract}

\begin{abstract}
The healthcare industry is evolving its service delivery, focusing on the quality of treatment to the patients. Thereis a necessity to monitor the health of the patients from the data analysis of these patients, aiming to detect possible diseases, among them, cardiovascular diseases. Within this context, this paper presents a e-Health intelligent system to analysis biological data of the patients and to detect possible cardio problems. The proposed system has three phases: (I) filtering the data; (II) useful information extraction; and, (III) analysis and classification to detect possible cardiovascular diseases. The experiments performed used a real database to show the capability of the proposed system to identify cardiovascular anomalies.
\end{abstract}

\section{Introdução}

Devido o grande avanço das tecnologias computacionais, temos diversas áreas de aplicações em expansão. Internet das Coisas (IoT), Computação em Nuvens, sensores de monitoramento, são exemplos de tecnologias utilizadas para melhorar e apoiar a demanda de outras áreas do conhecimento. Ao conectar estes dispositivos a Internet, tem-se uma ampla gama de aplicações que podem facilitar e auxiliar as mais diversas necessidades.

Com uma maior preocupação da saúde humana, uma área em expansão é a ehealth que tem como intuito dar suporte à medicina por meio de Tecnologias de Informação e Comunicação (TIC). Esta é uma das áreas mais promissoras e com maior impacto econômico, onde espera-se um crescimento entre \$1.1 a \$2.5 trilhões anualmente pela economia global de 2025, tendo um crescimento superior às demais aplicações [Al-Fuqaha et al. 2015]. Pela interação das Redes IoT com a tecnologia e-health, 
esperam-se sistemas capazes de monitorar, analisar e enviar informações sobre determinado paciente em tempo real. Com isso, a aplicação da computação à área médica vem colaborando para melhorar e definir um conjunto de novos processos para a automação na área de saúde. Entretanto, é necessário ter muito cuidado no processo de implantação de sistemas inteligentes por lidar com vidas humanas [Begg et al. 2007].

A grande demanda de cuidados médicos resulta na necessidade urgente de uma infraestrutura de monitoramento de saúde em tempo real para coletar dados biológicos de exames (periodicamente ou em tempo real), analisar estes dados dos pacientes e detectar possíveis doenças, visando aprimorar tratamento dos pacientes e reduzir os casos de alta gravidade [Mendis et al. 2011]. Dentre exames e sinais vitais importantes podemos citar o ECG (Eletrocardiograma), a pressão sanguínea, oxigenação, dentre outros. Adicionalmente, pode-se utilizar os dados biológicos monitorados de forma isolada ou em conjunto a fim de prever possíveis problemas à saúde dos pacientes [Zhao et al. 2018].

Um campo crítico que necessita de grande atenção é o relacionado a Doenças cardiovasculares (DCV), pois representam a principal causa de mortes no Mundo, onde estima-se que quase 18 milhões de mortes em 2016 devido a DCV sendo responsável $31 \%$ das mortes mundiais [Organization et al. 2016]. O alerta, a prevenção e o tratamento adequados podem reverter essa grave situação segundo a World Health Organizarion [Organization et al. 2016].

Métodos computacionais foram propostos para automatizar o processo de análise de ondas de eletrocardiograma (ECG), ajudando no processo de tomada de decisão dos médicos, porém poucos trabalhos relatam a detecção e classificação das anomalias referentes a estas ondas. Mesmo que tenham atingido desempenhos razoáveis, vários deles dependem de algoritmos computacionalmente complexos e podem ser muito custosos em termos de processamento e memória.

Dentro deste contexto, este artigo propõe um sistema e-Health inteligente para analisar os dados biológicos coletados dos pacientes e detectar possíveis complicações cardíacas. O principal objetico é classificar as ondas de ECG, com alta acurácia e rápida resposta, em diversos tipos de anomalias. No sistema proposto aplica-se três fases: (I) trata-se os dados através de filtros, melhorando a capacidade de distinção; (II) técnicas para extração das informações úteis são utilizadas para transformar o sinal original do ECG para um vetor de características significativas; e, (III) os dados são analisados e classificados utilizando Árvores de Decisão, Florestas Aleatórias, Máquina de vetores de suporte e Perceptron Multicamadas (MLP) para detectar doenças cardíacas.

O sistema proposto é avaliado a partir de experimentos que utilizam uma base de dados real [Moody and Mark 2001], onde foram avaliados o tempo de processamento dos dados e a acurácia na detecção de doenças. Os resultados mostram que o sistema proposto é capaz de classificar com alta acurácia 18 diferentes anomalias cardíacas, alcançando uma percentual de $98 \%$ de acurácia.

O restante deste artigo está organizado da seguinte forma. A Seção 2 discute vários trabalhos relacionados, levando em consideração a análise de dados cardíacos. A Seção 3 descreve a construção do blocos utilizados para detecção de anomalias e detalha o método proposto, enquanto a Seção 4 descreve os resultados obtidos através do método relatado. Finalmente, a Seção 5 conclui o artigo e apresenta trabalhos futuros. 


\section{Trabalhos Relacionados}

Nesta seção serão descritos os trabalhos relacionados que compreendem a classificação de anomalias e diagnóstico de doenças cardíacas através da onda ECG, com diferentes abordagens.

Um sistema de monitoramento de pacientes é apresentado por Kiranyaz, Ince e Gabbouj (2016) . Este trabalho utiliza uma Rede Neural Convolucional (CNN) de uma dimensão para classificação de doenças cardíacas através do ECG. Os dados são analisados separadamente através de redes CNN diferentes, criando assim uma rede personalizada para cada paciente.

Mesmo com bons resultados, este trabalho limita-se na detecção de apenas duas anomalias cardíacas, sendo elas batimento ventriculares prematuros e contrações ventriculares prematuras.

Oresko et. al desenvolveu uma aplicação para smartphones capaz de classificar pontos de importância e doenças cardíacas. Para detecção destes pontos, o trabalho utilizou um algoritmo baseado em [Hamilton and Tompkins 1986] que utiliza um limiar adaptativo. Quanto a classificação dos sinais, Oresko utilizou uma Rede Perceptron de multicamadas (MLP) que como entrada tem-se um sinal de complexo QRS com 51 amostras de segmentos com tempos de $150 \mathrm{~ms}$. A saída da rede MLP é então associada a um dos problemas cardíacos analisados no escopo do trabalho. Para a implementação desta rede utilizou-se o MATLAB e para validação dos resultados, utilizou-se a base de dados MIT-BIH [Moody and Mark 2001].

Este trabalho também apresentou bons resultados para as anomalias analisadas, porém assim como os outros, tem uma limitação na quantidade destas anomalias, trabalhando apenas com quatro, que são: Bloqueio do ramo direito do feixe de His, contração ventricular prematura, batimento cardíaco estimulado por um marcapasso e fusão destes batimentos com batimentos normais.

Em Carrera et al. propõe-se um sistema de monitoramento de ECG, capaz de modelar os batimentos normais de um paciente através de processos de linguagem natural, onde os padrões não conhecidos por este modelo são classificados como anormais. Através de transformações lineares e um conjunto de regras, um dicionário é auto adaptado para os usuários. Através deste modelo, os batimentos anormais são detectados através da distancia entre os subespaços referentes a estes eventos, afirmando como anormal as distâncias fora deste padrão.

Este trabalho apresenta um esquema complexo com ótimos resultados médios na detecção de anormalidade, porém demonstra uma alta variação de precisão de acordo com a alteração da frequência cardíaca, com resultados de falso positivos baixos para determinados pacientes, porém altos para os demais. Além destes fatores, este trabalho relaciona somente duas classes de anomalias, classificando os pacientes com batimentos cardíacas normais e anormais, sem especificar qual a classe da anormalidade.

É apresentado em Xu et al. 2018 um sistema capaz realizar a detecção de arritmias e a análise de dados em larga escala, chamado ECGLens, integrando um sistema de classificação de batidas do coração baseado em redes neurais convolucionais. Para este modelo, foram considerados quatro diferentes anomalias, Ritmo sinusal, batimento ventricular 
prematuro, Atividade Ectópica Supraventricular e fusão sinusal e ventricular prematura. No processo de detecção de anomalias, este sistema implementa um modelo automático de série temporal baseada em modelos multi escala de redes neurais convolucionais. $\mathrm{O}$ processo é realizado em três fases, primeiramente utiliza-se técnicas de transformações espectrais e redução por amostragem com o intuito de minimizar os ruídos e melhorar as entradas. Após este processo, os dados são submetidos a um algoritmo convolucional de três camadas seguido por uma camada com a função de ativação ReLU e outra maxpooling. Em um último estágio, o dado é concatenado, e ocorre o envio para duas camadas convolucionais, cada uma seguida por uma outra camada ReLU e outra maxpooling, uma camada totalmente conectada e uma camada probabilística softmax.

Este trabalho propõe um sistema complexo de análise de anomalias em uma onda ECG, porém como resultado, apresenta uma acurácia intermediária de detecção dos batimentos cardíacos. Observa-se também que esta proposta analisa somente quatro diferentes anormalidades.

Tabela 1. Comparação das análises realizadas nos trabalhos relacionados.

\begin{tabular}{lcccc}
\hline \multicolumn{1}{c}{ Trabalho } & $\begin{array}{c}\text { Quantidade de Anomalias } \\
\text { detectadas }\end{array}$ & Custo Computacional & $\begin{array}{c}\text { Completude da Base } \\
\text { de Teste }\end{array}$ & Numero de atributos \\
\hline [Kiranyaz et al. 2016] & 2 & Alto & Parcial & Parte da Onda \\
[Oresko et al. 2010] & 4 & Baixo & Completa & 6 \\
[Carrera et al. 2019] & 2 & Baixo & Completa & Parte da Onda \\
[Xu et al. 2018] & 4 & Alto & Completa & 4 \\
Este trabalho & 18 & Baixo & Completa & 10 \\
\hline
\end{tabular}

Os resumos das principais características de cada trabalho apresentado nesta seção pode ser observado através da Tabela 1, onde destaca-se a quantidade de anomalias detectadas em cada trabalho, seu custo computacional, a completude do trabalho em analisar a base de dados e o número de atributos considerados para a classificação.

\section{Proposta}

Para realizar a classificação das anomalias em um ECG, é necessário a construção de um modelo de análise da onda que pode ser divididos em três principais processos. Primeiramente os dados recebidos em forma de onda devem ser tratados através de filtros capazes remover artefatos indesejados, como ruídos elétricos ou musculares.

Em um segundo passo, as informações úteis presentes na onda devem ser extraídas através de processos de detecção destes pontos de importância. Por último, as informações extraídas são utilizadas para diferenciar e detectar as diferentes anomalias existentes no exame dos pacientes.

\subsection{Filtragem da Onda e Detecção dos Pontos de Importância}

A fim de analisar o ECG para detecção dos pontos de importância deve-se inicialmente realizar a filtragem dos dados recolhidos, uma vez que ruídos são facilmente introduzidos em um ECG através de circuitos de alta impedância de entrada e com um alto ganho para detecção de ondas de baixas voltagens produzidas pelo coração [Metshein et al. 2017]. Temos também o ruído introduzido através da rede elétrica local, da atividade muscular e da respiração que é uma das principais causas da variação da linha de base no ECG.

Em Moreira et al. apresenta-se os processos necessários para filtragem dos artefatos indesejados da onda ECG, utilizando para isto, filtros com base em Transformadas 
Discretas de Wavelet, que preparam a onda para, na etapa seguinte, realizar a extração dos segmentos de importância.

Com o sinal de ECG já filtrado, os blocos de interesse são então gerados, sendo compostos pelo complexo QRS e uma onda T. Utiliza-se os blocos QRS devido sua grande importância para detecção de doenças cardíacas. Para geração destes blocos, utilizou-se o esquema de médias móveis de acordo com Elgendi (2016). Este esquema utiliza dois períodos de tempo: $W_{1}$, a duração aproximada do evento de interesse, e $W_{2}$, a duração aproximada do ciclo completo de um batimento cardíaco. As médias móveis $\mathrm{MA}_{\text {evento }}$ e $\mathrm{MA}_{\text {ciclo }}$ são então computadas através dos tempos $W_{1}$ e $W_{2}$ utilizando-se a Equação 1 e Equação 2, tendo $n$ como índice de cada ponto dos $N$ pontos de dados e y [n] o valor atual.

$\mathrm{MA}_{\text {evento }}[n]=\frac{1}{W_{1}}\left(y\left[n-\frac{W_{1}-1}{2}\right]+y\left[n-\frac{W_{1}-3}{2}\right]+\cdots+y\left[n+\frac{W_{1}-3}{2}\right]+y\left[n+\frac{W_{1}-1}{2}\right]\right)$

$\mathrm{MA}_{\text {ciclo }}[n]=\frac{1}{W_{2}}\left(y\left[n-\frac{W_{2}-1}{2}\right]+y\left[n-\frac{W_{2}-3}{2}\right]+\cdots+y\left[n+\frac{W_{2}-3}{2}\right]+y\left[n+\frac{W_{2}-1}{2}\right]\right)$

Todas as regiões que $y[n]$ está contido em $\mathrm{MA}_{\text {evento }} \geq \mathrm{MA}_{\text {ciclo }}$ são então consideradas como pontos de interesse, sendo calculadas através dos vetores de médias móveis. Os valores de limiar $\mathrm{MA}_{\text {evento }}$ e $\mathrm{MA}_{\text {ciclo }}$ podem ser ajustados de acordo com quão ruidoso encontra-se o sinal através da adição de um deslocamento, normalmente, proporcional à média do sinal. Os melhores valores de períodos referentes aos complexos QRS, para detecção dos blocos QRS, foram calculados de acordo com a base de dados MIT-BIH. Para cada valor, calcula-se a média móvel $\mathrm{MA}_{\mathrm{QRS}}$ do evento e a média móvel $\mathrm{MA}_{\text {batimento }}$ do ciclo, seguindo a Equação 1 e Equação 2. Em alguns casos, outros eventos, como as ondas $\mathrm{P}$ e T, podem ser detectados junto com o complexo QRS, para evitar estes erros, regiões abaixo dos valores $W_{1}$ ms são descartadas.

Com a detecção dos complexos QRS concluída, procura-se então detectar os picos $\mathrm{Q}, \mathrm{R}$ e S. Tendo como maior valor do vetor do complexo, atribuído ao pico $\mathrm{R}$, sendo o pico com maior amplitude do sinal. Os valores máximos da onda à esquerda e direita a partir do pico R, em um sinal quadrático, são atribuídas aos picos Q e R respectivamente. Uma vez já detectado o complexo QRS, este sinal é então removido da onda original, zerando os seus valores correspondentes no vetor, para prosseguir com a detecção dos pontos fiduciais P e T. Após isto, a onda contém apenas os sinais ainda não identificados. Um resultado típico pode ser visto na Figura 1.

Foram também realizados testes para determinar os melhores valores para os intervalos $W_{1}$ e $W_{2}$ da onda T, levando em considerando a base de dados MIT-BIH. A média móvel relacionada ao evento $\mathrm{MA}_{\mathrm{T}}$ e a média móvel relacionada ao ciclo das ondas $\mathrm{MA}_{\text {batimento }}$ são então calculadas para o novo evento, aplicando a Equação 1 e Equação 2 para o sinal sem o complexo QRS. Remove-se então a onda T do sinal, deixando apenas a onda $\mathrm{P}$ e alguns ruídos. O maior valor à esquerda do pico $\mathrm{R}$ é considerado o pico $\mathrm{P}$. 


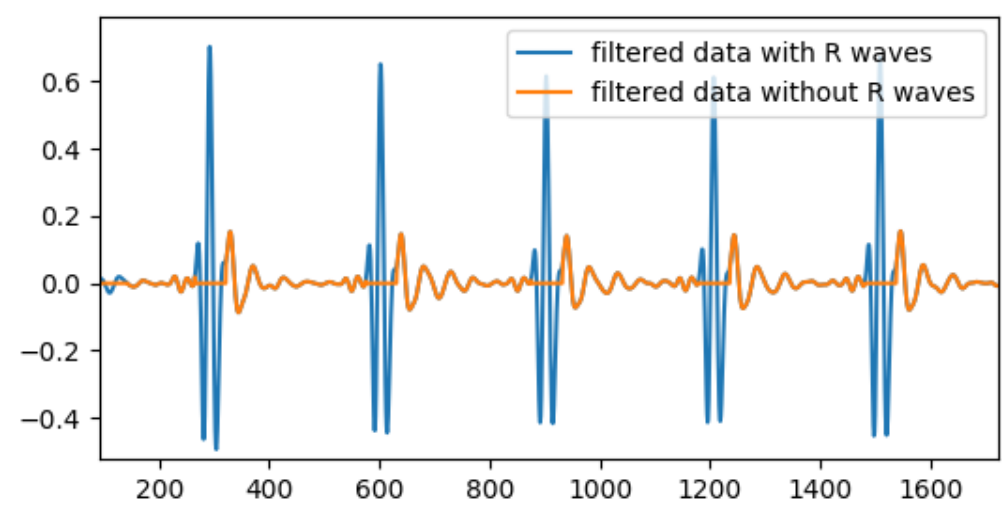

Figura 1. Sinal após remoção do complexo QRS.

Como passo de melhoria realiza-se uma busca dos picos reais das ondas $\mathrm{P}$ e $\mathrm{T}$, entre os pontos existentes com base no espaço de ocorrência de cada onda, pelos valores regulares das distâncias entre as ondas PR e entre as ondas RT em um ECG, descartando possíveis valores classificados erroneamente. Em um alcance dentro dessa distância, podemos definir quais pontos são realmente reais, considerando pontos que estão fora deste intervalo como pontos de erro. Realiza-se esta busca somente a partir dos picos $\mathrm{R}$, pois as ondas $\mathrm{P}$ e $\mathrm{T}$ podem ocorrer em pontos que não são detectados uma onda $\mathrm{R}$, em ondas anômalas.

Como resultado desta fase, produz-se um sinal com todos os picos de importância detectados, visto na Figura 2, que serão então utilizados na fase de classificação. Este pontos serão então agrupados para então serem utilizados pelos algoritmos de classificação. Cada parte da onda representa um período médio dos batimentos do paciente, possibilitando um diagnóstico segmentado.

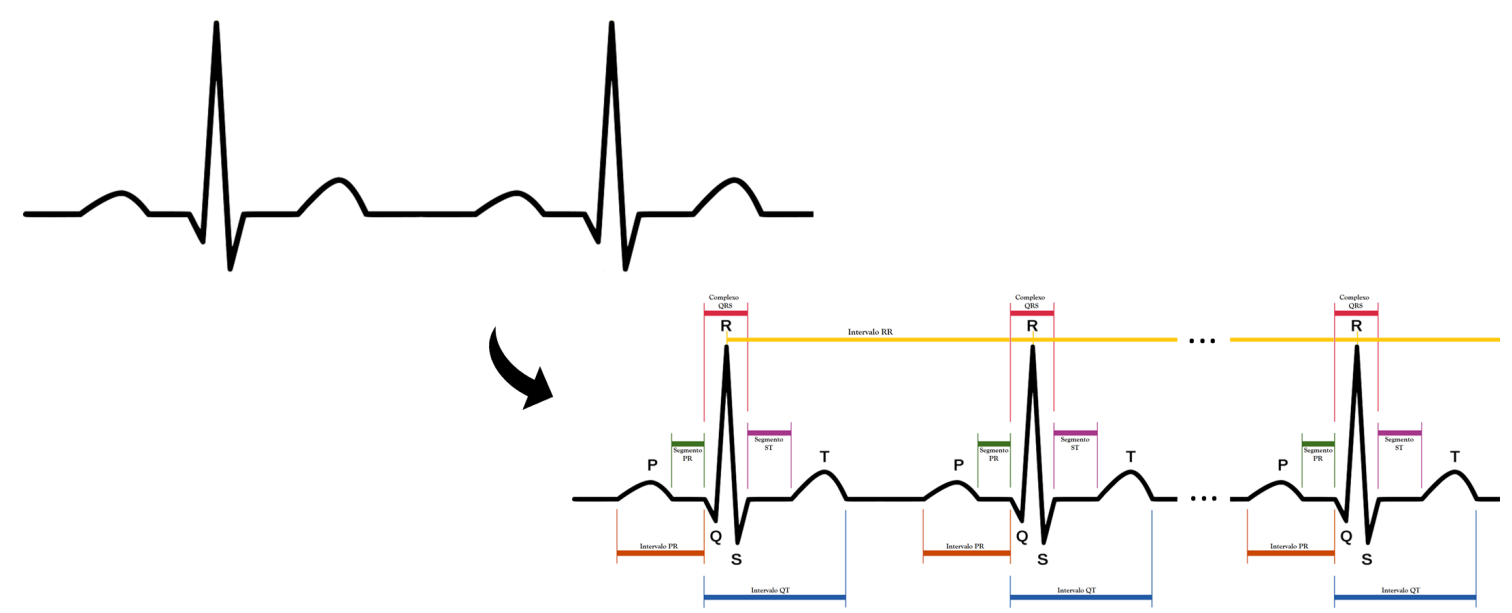

Figura 2. Onda com pontos fiduciais detectados.

A próxima fase, após a detecção dos pontos fiduciais, é a utilização destes pontos para formação dos blocos de interesse.

\subsection{Blocos de interesse}

Para classificar a onda, definiu-se um sistema de blocos. Cada bloco é formado por um conjunto de pontos de importância, referentes a um período da onda. Entre os pontos de 
importância recebidos da etapa anterior, têm-se os pontos $\mathrm{P}, \mathrm{Q}, \mathrm{R}, \mathrm{S}$ e T. Cada um destes pontos têm informações da amplitude da onda. A partir dos pontos fiduciais, também são formados segmentos de importância, que representam a distância em amostras entre os pontos. Estes segmentos são definidos segundo os principais segmentos cardíacos existentes, segmento PR, RR, QT, ST e QRS. Desta forma cada bloco pode ser representado por um período que compreende os pontos fiduciais e os segmentos cardíacos entre uma onda $\mathrm{P}$ à $\mathrm{T}$, como pode ser observado na Figura 3.

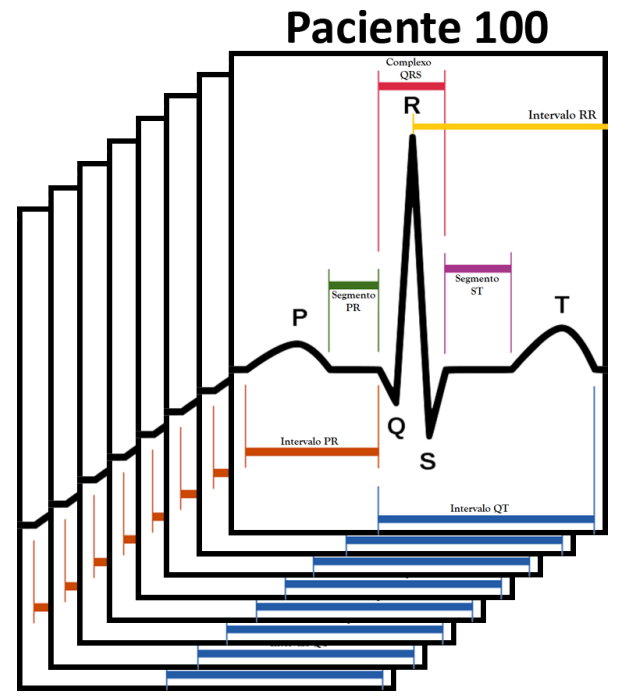

Figura 3. Blocos formados pelos períodos e segmentos compreendidos entre uma onda $P$ à $T$

O ECG pode ser representado por um conjunto de blocos de interesse. Cada bloco pode ser analisado separadamente ou em conjunto. Esta abordagem possibilita, agora, uma análise não somente da onda completa de ECG, mas como frações de onda. Este processo facilita, principalmente, na aquisição de dados, visto que registros com menos tempo de duração podem ser incorporados ao sistema facilmente. Como também auxilia o diagnóstico em um menor período de tempo, visto que remove a necessidade de uma onda completa para a análise, bastando assim apenas um único bloco do paciente para iniciar o processo de classificação.

Como um último passo, estes blocos são então enviados para as máquinas de aprendizagem, onde realiza-se a classificação, baseando-se nos atributos contidos no bloco recebido.

\subsection{Classificação de Anomalias}

Para realizar a classificação das possíveis anomalias em um bloco, serão utilizados algoritmos de aprendizagem de máquinas, Árvores de Decisão, Florestas Aleatórias, SVM e métodos de aprendizagem profunda, como Perceptron multicamadas. As doenças são classificadas em relação aos 10 atributos de entrada relacionados a formação do bloco, picos P, Q, R, S, T além dos segmentos e intervalos PR, RR, QT, ST e QRS, todos estes foram propostos por este trabalho. Como entrada para esta fase, pode-se receber, já preprocessado, um bloco isolado de ECG, o conjunto de blocos de um determinado paciente ou um conjunto de clusters formado por blocos agrupados de diferentes pacientes. Estes conjuntos de dados constituem a entrada para os algoritmos de aprendizagem, Figura 4. 


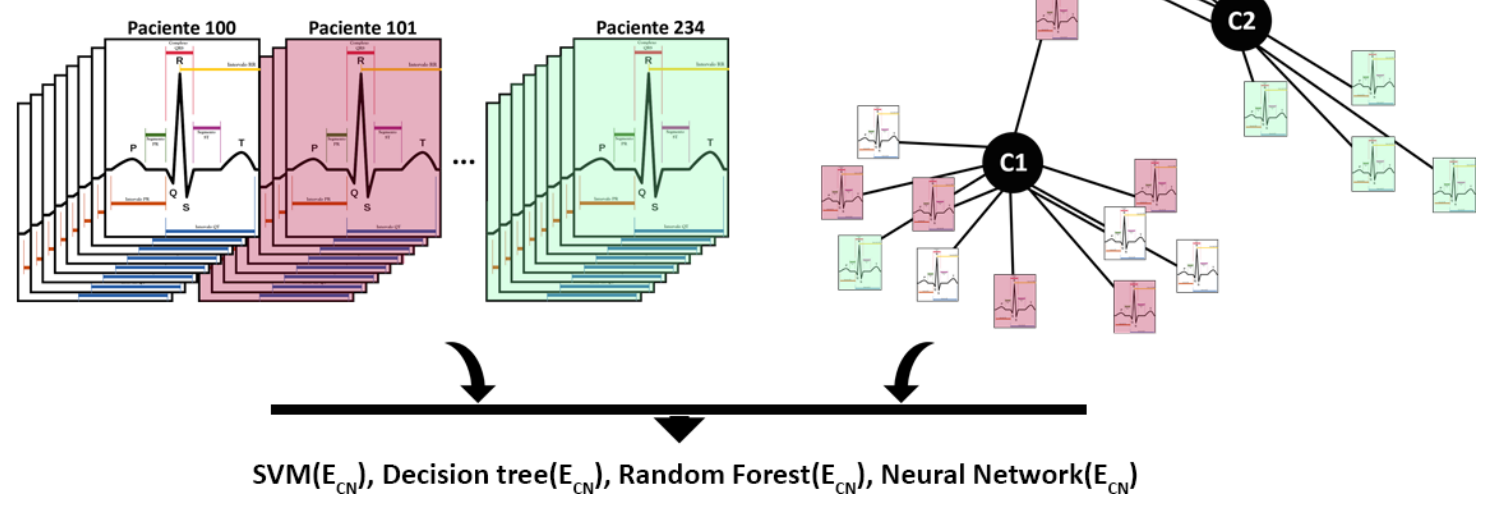

Figura 4. Classificação dos blocos ECG pelos algoritmos de aprendizagem de máquina.

No caso de receber somente um bloco, o objetivo do sistema é classificá-lo como um trecho saudável ou com anomalias, sendo utilizado para análises pontuais ou para reanálise de trechos específicos, este trecho é então re-integrado ao seu contexto para analise do especialista caso necessário.

Para um conjunto de blocos de um único paciente, classificam-se as anomalias deste paciente e se lista todos os blocos com irregularidades, desta forma, cria-se uma máquina especialista nos sinais e problemas do paciente, com isto, pode-se obter ótimos resultados em um exame contínuo de observação. Este cenário é indicado para pacientes que necessitam de uma longa observação pois longos trechos de ECG são difíceis de observar, pelo método proposto, este trecho é facilmente classificado, gerando um retorno menor para a observação do especialista.

Para entradas de um conjunto de blocos de diferentes pacientes, gera-se uma máquina com objetivos amplos, de forma que pode-se ter um melhor resultado na classificação de doenças em geral. Este cenário é indicado para detecção de novas anomalias em diferentes pacientes com uma grande variedade e quantidade de sinais.

Para os dois cenários apresentados e propostos por este trabalho, ambas as máquinas geram resultados capazes de detectar e informar especificamente as anomalias, ou seja, qual a doença está atrelada ao sinal analisado. No próximo capitulo serão apresentados os resultados obtidos através da aplicação da proposta aqui apresentada. Primeiramente verificar o processo de criação e implementação do sistema e depois os resultados quantitativos em comparação com outros trabalhos da área.

\section{Avaliação de Desempenho}

Esta seção descreve os experimentos realizados para avaliar o sistema e-Health proposto em relação a análise de dados e detecção de problemas cardíacos. A Seção 4.1 apresenta a metodologia aplicada durante a avaliação da proposta, enquanto que a Seção 4.2 analisa os resultados numéricos dos experimentos realizados. 


\subsection{Metodologia}

O desempenho do sistema proposto foi avaliado utilizando o banco de dados real de arritmias $M I T-B I H$, e comparado com o sistema apresentado na referência [Oresko et al. 2010]. O MIT-BIH é composto por quarenta e oito trechos de gravações de ECG, com meia hora cada, de dois canais. Os registros foram obtidos de 47 indivíduos estudados pelo Laboratório de Arritmias do Hospital Beth Israel, em Boston, entre 1975 e 1979 que foram coletados de uma população mista de pacientes internados (cerca de 60\%) e ambulatoriais (cerca de 40\%) no Hospital Beth Israel e digitalizados em 360 amostras por segundo em cada canal e com resolução de 11 bits em um intervalo de dez (10) $\mathrm{mV}$. Dois ou mais cardiologistas anotaram independentemente os registros para cada batimento cardíaco (aproximadamente 110.000 anotações) incluídos no banco de dados, onde discordâncias foram resolvidas para obter legibilidade.

Durante os experimentos foram analisados todos os trechos de ECG disponíveis no MIT-BIH com as seguintes métricas de avaliação: Acurácia (percentual de acertos em relação ao montante de dados) e tempo de análise da técnica escolhida. Da mesma forma, foram avaliadas as técnicas de aprendizado de máquina Árvore de Decisão, Florestas Aleatórias e SVM implementadas com as bibliotecas python de aprendizagem de máquina scikit-learn [Pedregosa et al. 2011] e MLP implementada utilizando a Biblioteca TensorFlow [Abadi et al. 2015].

Para classificação dos sinais levou-se em conta 19 diferentes classes de eventos cardíacos, com 18 anomalias [Moody and Mark 2001]: Batimento Normal, Bloqueio do ramo esquerdo do feixe de His, Bloqueio do ramo direito do feixe de His, Batimento Prematuro Atrial, Contração Ventricular Prematura, Fusão de batimentos ventriculares e normais, Batimentos Nodais Prematuros, Batimentos atriais prematuros, Batimento supraventricular prematuro ou ectópico, Batimento de escape ventricular, Batimento de escape nodal, Batimento estimulado, Bloqueio atrioventricular do primeiro grau à esquerda ou direita, Onda de fibrilação ventricular, Batimento de escape atrial, Batimento de escape Supraventricular, Onda P não conduzida (APB com clock), Fusão de batimento ritmado e normal, Contração ventricular prematura R-em-T.

\subsection{Resultados}

Para avaliação da classificação dos pacientes em relação a anomalias cardíacas, recebese como entrada os blocos de importância do sinal ECG. A partir disso, cada bloco é classificado utilizando os algoritmos de árvores de decisão, floresta aleatória, SVM e MLP. Estes blocos foram avaliados em dois principais cenários.

O primeiro cenário realiza a classificação de um conjunto de blocos de um único paciente, ou seja, todos os blocos de um deste paciente são recebidos e classificados de acordo com suas anomalias. Em segundo cenário os blocos de entrada fazem parte de um conjunto de blocos de diferentes pacientes.

Para o treinamento dos algoritmos de classificação utilizou-se a técnica de Validação Cruzada (Cross-validation) [Wong 2015], onde 67\% dos dados são utilizados para o treinamento dos algoritmos e os $33 \%$ restantes dos dados como entrada para os testes de classificação. Diferentes configurações foram utilizadas para múltiplos testes dos algoritmos de aprendizagem, uma média de 33 execuções de cada algiritmo foi tomada. A 
combinação das seguintes configurações, para cada um dos algoritmos, pode ser observada na Tabela 2

Tabela 2. Hiperparâmetros dos Algoritmos de Aprendizagem.

\begin{tabular}{|c|c|c|c|}
\hline Algoritmo de Aprendizagem & Hiperparâmetros & Valores & Valores com Melhores Resultados \\
\hline \multirow{5}{*}{ Árvores de Decisão } & Critério & Gini, Entropy & Entropy \\
\hline & Splitter & Best, Random & Best \\
\hline & Min. Samples Split & $\frac{m s s}{10} ; m s s \in[1,10], m s s \in \mathbb{N}$ & 0.1 \\
\hline & Min. Samples Leaf & $\frac{m s s}{10} ; m s s \in[1,5], m s s \in \mathbb{N}$ & 0.1 \\
\hline & $\mathrm{N}^{\circ}$ Max. recursos & $1,2,3,4,5,6,7,8,9,10$, auto, sqrt, $\log 2$, None & 8 \\
\hline \multirow[t]{7}{*}{ Floresta Aleatória } & Critério & Gini, Entropy & Entropy \\
\hline & Splitter & Best, Random & Best \\
\hline & Min. Samples Split & $\frac{m s s}{10} ; m s s \in[1,10], m s s \in \mathbb{N}$ & 0.7 \\
\hline & Min. Samples Leaf & $\frac{m s s}{10} ; m s s \in[1,5], m s s \in \mathbb{N}$ & 0.4 \\
\hline & $\mathrm{N}^{\circ}$ Max. recursos & $1,2,3,4,5,6,7,8,9,10$, auto, sqrt, $\log 2$, None & 4 \\
\hline & Estimadores & 10,20 & 20 \\
\hline & $\mathrm{C}$ & $0.25,0.50,0.75,1$ & 1 \\
\hline \multirow{3}{*}{ SVM } & Kernel & linear, poly, sigmoid, rbf & rbf \\
\hline & Grau & $1,2,3$ & - \\
\hline & coef0 & $0.25,0.50,0.75,1$ & 0.25 \\
\hline \multirow{4}{*}{ MLP } & $\begin{array}{l}\mathrm{N}^{\circ} \text { de Neurônios - } \\
\text { Primeira Camada }\end{array}$ & $128,256,512,1024,2048$ & 1024 \\
\hline & $\begin{array}{l}\mathrm{N}^{\circ} \text { de Neurônios - } \\
\text { Segunda Camada }\end{array}$ & $128,256,512,1024,2048$ & 1024 \\
\hline & Taxa de Aprendizado & 0.001 & 0.001 \\
\hline & Otimizador & Adam [Kingma and Ba 2014], Gradiente Descendente & Adam [Kingma and $\mathrm{Ba} 2014]$ \\
\hline
\end{tabular}

Para árvores de decisão, os hiperparâmetros utilizados foram o critério Gini e Entropia, medida para mensurar a qualidade da divisão dos ramos. Spliter define a estratégia de divisão em cada nó, melhor ou aleatória, ajustando a melhor divisão ou de modo aleatório, sem critério. O mínimo de amostras para divisão define o número mínimo de amostras que são requeridas para dividir um nó interno.

A quantidade mínima de amostras nas folhas define a quantidade de amostras que são requeridas para ser um nó folha. Por último, o número máximo de recursos, é considerado ao procurar a melhor divisão, este valor pode variar em um inteiro de 1 a 10, que considera uma quantidade fixa de valores, SQRT, ou seja, a raiz quadrada da quantidade de características, $\log 2$, definindo o número máximo como o $\log _{2}$ e nenhum para o número máximo igual à quantidade de características.

Os hiperparâmetros considerados para Floresta Aleatória compreendem o conjunto de parâmetros das árvores de decisão, com a adição do número de estimadores que define a quantidade de árvores em uma floresta, foram utilizadas 10 e 20 árvores.

No SVM, utilizou-se o parâmetro de erro C, definindo um valor para evitar classificar erroneamente cada exemplo de treinamento, através de uma margem no hiperplano gerado. Os kernels utilizados foram o linear, polinomial, sigmoide e rbf. Para definir o grau do kernel polinomial, utiliza-se os de $1^{\circ}, 2^{\circ}$ e $3^{\circ}$ grau. Como termo independente nas funções polinomiais e sigmoides, utilizou-se o parâmetro coef0, com os valores 0.25 , $0.50,0.75$ e 1 .

Nas MLP foram utilizadas duas camadas intermediárias com o número de neurônios igual à combinação dos valores $128,256,512,1024,2048$. A taxa de aprendizado da rede foi definida como 0.001 e os otimizadores Adam e o Gradiente Descendente foram utilizados na avaliação da rede.

Na classificação do primeiro cenário, onde todos os blocos de treinamento e classificação pertencem apenas ao paciente que terá seus blocos classificados, os resultados obtidos podem ser observados na Tabela 3 . 
Tabela 3. Resultado dos Algoritmos de Aprendizagem para as 19 diferentes classes de eventos cardíacos, com 18 anomalias em um cenário de análise geral.

\begin{tabular}{ll}
\hline Algoritmos de Aprendizagem & Acurácia \\
\hline Árvore de Decisão & 0.9530 \\
Florestas Aleatória & 0.9502 \\
SVM & 0.9818 \\
MLP & 0.9878 \\
\hline
\end{tabular}

Este cenário pode ser utilizado, por exemplo, para monitoramento de pacientes separadamente, onde cada paciente tem seu próprio escopo e uma máquina especialista para classifica-lo, obtendo um resultado de até $98 \%$ de acurácia na classificação das anomalias cardíacas. Por meio desta classificação, pode-se por exemplo, realizar uma observação prolongada deste paciente e retornar apenas os períodos com maior quantidade de anomalias detectadas, com o objetivo de monitoramento ao invés de classificação de novas doenças. Neste cenário, observa-se que as máquinas de aprendizado tiveram um bom resultado em geral, visto que foram treinadas de forma especialista, com um menor número de classes possíveis, não apresentando um grande desvio de resultado.

Para o segundo cenário, de classificação dos blocos em um conjunto de outros blocos, não necessariamente de um mesmo pacientes, as médias dos resultados obtidos para estas anomalias podem ser observadas na Tabela 4.

Tabela 4. Resultado dos Algoritmos de Aprendizagem para as 19 diferentes classes de eventos cardíacos, com 18 anomalias em um cenário de análise especialista

\begin{tabular}{ll}
\hline Algoritmos de Aprendizagem & Acurácia \\
\hline Árvore de Decisão & 0.7122 \\
Florestas Aleatórias & 0.7094 \\
SVM & 0.8820 \\
MLP & 0.9640 \\
\hline
\end{tabular}

Este cenário pode ser utilizado, por exemplo, em um grande conjunto de dados onde todos os pacientes fazem parte de um único escopo. Neste caso, um paciente com doenças ainda não conhecidas pode ser diagnosticado sem a necessidade de uma máquina especialista ou ser usada em conjunto para descobrir novas doenças em pacientes pertencentes ao outro cenário. Constrói-se uma máquina generalista, capaz de detectar novas anomalias em diferentes pacientes. Diferentemente do cenário anterior, neste observa-se uma grande variação dos resultados entre as máquinas utilizadas, mesmo obtendo bons resultados, visto que com o maior número de classes envolvidas, a técnica de MLP apresentou uma melhor generalização. Os melhores resultados foram obtidos através das configurações apresentadas na Tabela 2 na coluna referente aos valores com melhores resultados, para cada um dos algoritmos testados anteriormente.

Apesar das diferentes abordagens, em ambos os casos os blocos dos pacientes podem ser classificados um a um, provendo um ambiente com resultados imediatos, porém os blocos são disponibilizados em faixas de exame para que o especialista possa avalialos.

Para realizar a comparação dos resultados obtidos neste trabalho com os resultados 
de Oresko et al. e Xu et al., também utilizou-se os algoritmos de classificação em um escopo de apenas quatro doenças. Onde Oresko levou em consideração as anomalias de Bloqueio do ramo direito do feixe de His, Contração ventricular prematura, ritmos estimulados e fusão de ritmos estimulados com batimentos normais. E Xu as anomalias Ritmo sinusal, batimento ventricular prematuro, Atividade ectópica Supraventricular e fusão sinusal e ventricular prematura. Estes resultados são descritos na Tabela 5 e levam em consideração um cenário composto por sequências de blocos de diferentes pacientes.

\section{Tabela 5. Resultado dos Algoritmos de Aprendizagem para as 4 diferentes ano- malias por bloco}

\begin{tabular}{cll}
\hline \multicolumn{2}{l}{ Algoritmos de Aprendizagem } & Acurácia \\
\hline & Árvore de Decisão & 0.7927 \\
& Florestas Aleatórias & 0.7886 \\
& SVM & 0.9218 \\
& MLP (Adam) & 0.9825 \\
\hline$[$ Oresko et al. 2010] & MLP & 0.93 \\
\hline$[$ Xu et al. 2018] & CNN & 0.91 \\
\hline
\end{tabular}

Pode-se observar um ganho de até $7 \%$ na classificação das anomalias, se comparado ao cenário de classificação com blocos de diferentes pacientes. Estes resultados devem-se a estratégia de divisão dos blocos de interesse e a utilização do otimizador adaptativo Adam [Kingma and Ba 2014] para aprendizagem profunda através da plataforma para aprendizagem profunda TensorFlow.

Em comparação com os demais trabalhos relatados na literatura, obteve-se um ótimo resultado, levando em consideração o método de MLP. Em comparação com Oresko et al., tem-se um ganho de até 5\% na detecção de anomalias, que corresponde a uma melhora média a 232 batimentos classificados corretamente por hora em um único paciente. Quanto a Xu el tal. a melhora na classificação das anomalias é de 7\%, até 323 batimentos por hora em um único paciente, esta melhoria deve-se principalmente à estratégia de blocos proposta neste trabalho, utilizando-se 10 atributos diferentes para a detecção.

Este trabalho demonstra o alto grau de precisão deste sistema, se comparado até com sistemas com apenas duas classes de classificação, como Kiranyaz et al. e Carrera et al., que relatam uma acurácia máxima de 97,68\%. Sabe-se que para o resultado obtido, a parcela negativa foi influenciada pela quantidade de amostras relacionadas a certas doenças que estavam abaixo do necessário para o treinamento da maquina utilizada. Alguns outros casos demonstram erros em relação a proximidade dos sinais saudáveis com os que contém anomalias. Porém, devido ao baixo valor de erro, o sistema é totalmente utilizável, já que os alertas gerados para análise médica em sua grande maioria é correto e com ótima precisão.

Em relação ao tempo decorrido para classificação das anomalias dos pacientes, no pior cenário, com a classificação de todos os pacientes, em média 650,000 amostras e 2314 complexos QRS e utilizando o algoritmo com maior performance de classificação, uma rede MLP, tem-se um resultado em apenas 2.8 segundos para classificação de todos os blocos existentes. Este resultado demonstra a capacidade de tal sistema ser utilizado em situações de tempo real, visto que para gerar 2314 blocos, um ser humano leva em média 33 minutos. 
Os resultados foram obtidos tanto para a classificação dos pontos fiduciais de um ECG quanto a classificação destes pontos a partir de uma estratégia de separação por bloco. Cada bloco foi classificado em um conjunto de blocos do mesmo paciente ou de pacientes diferentes, apresentando assim as qualidades de cada abordagem. Também observou-se o tempo necessário para que o método seja utilizado em sistemas de tempo real. No próximo capítulo, apresenta-se as conclusões do trabalho e os trabalhos futuros.

\section{Conclusão e trabalhos futuros}

Devido ao grande número de mortes por doenças cardíacas, tornou-se importante ter uma preocupação extra para detectar essas doenças. A maneira mais comum é pelo eletrocardiograma (ECG) dos pacientes e de maneira visual. Para isso, é crucial detectar os pontos de importância de um ECG e classificar estes pontos em relação as possíveis anomalias.

Neste trabalho, propõe-se um sistema e-health responsável por detectar características de importância em um ECG e formar uma representação que é então utilizada para classificação de anomalias cardíacas por algoritmos de aprendizagem de máquina. A proposta foi avaliada utilizando a base de dados MIT-BIH que contém trechos de exames ECG reais. A proposta teve um bom desempenho tanto na deteç̧ão dos pontos de importância quanto à classificação das anomalias em relação a outros trabalhos importantes nesse domínio.

Como trabalhos futuros, pretende-se estender o sistema proposto para que este seja capaz de, a partir da estrutura base de análise e detecção de doenças apresentada, considerar outros exames (e consequentemente alguns outros dados biológicos) e detectar diversos tipos de doenças. Adicionalmente, pretende-se adicionar aspectos de big data, a fim de dar suporte a um grande volume de dados biológicos de diversos pacientes.

\section{Agradecimentos}

Os autores gostariam de agradecer a FUNCAP, CAPES e CNPq pelo apoio financeiro.

\section{References}

\section{Referências}

Abadi, M., Agarwal, A., Barham, P., et al. (2015). TensorFlow: Large-scale machine learning on heterogeneous systems. Software available from tensorflow.org.

Al-Fuqaha, A., Guizani, M., Mohammadi, M., Aledhari, M., and Ayyash, M. (2015). Internet of things: A survey on enabling technologies, protocols, and applications. IEEE communications surveys \& tutorials, 17(4):2347-2376.

Begg, R., Lai, D. T., and Palaniswami, M. (2007). Computational intelligence in biomedical engineering. CRC Press.

Carrera, D., Rossi, B., Fragneto, P., and Boracchi, G. (2019). Online anomaly detection for long-term ecg monitoring using wearable devices. Pattern Recognition, 88:482492.

Elgendi, M. (2016). Terma framework for biomedical signal analysis: An economicinspired approach. Biosensors, 6(4):55. 
Hamilton, P. S. and Tompkins, W. J. (1986). Quantitative investigation of qrs detection rules using the mit/bih arrhythmia database. IEEE transactions on biomedical engineering, (12):1157-1165.

Kingma, D. P. and Ba, J. (2014). Adam: A method for stochastic optimization. arXiv preprint arXiv:1412.6980.

Kiranyaz, S., Ince, T., and Gabbouj, M. (2016). Real-time patient-specific ecg classification by 1-d convolutional neural networks. IEEE Transactions on Biomedical Engineering, 63(3):664-675.

Mendis, S., Puska, P., Norrving, B., Organization, W. H., et al. (2011). Global atlas on cardiovascular disease prevention and control. Geneva: World Health Organization.

Metshein, M., Annus, P., Land, R., Krivošei, A., Ojarand, J., Aabloo, A., and Min, M. (2017). Variation of cardiac and respiratory waveform on human thoraxin the case of inductive coupling. In EMBEC \& NBC 2017, pages 671-674. Springer.

Moody, G. B. and Mark, R. G. (2001). The impact of the MIT-BIH arrhythmia database. IEEE Engineering in Medicine and Biology Magazine, 20(3):45-50.

Moreira, D. A., Chaves, L. G., Lima, B. A., Almeida, T. P., Gomes, R. L., Celestino, J., and Neto, A. J. (2018). Detecção de pontos fiduciais em eletrocardiogramas para sistemas de saúde. II Workshop de Computação Urbana (CoUrb), 2018, Campos do Jordão. Simposio Brasileiro de Redes de Computadores e Sistemas Distribuidos (SBRC).

Oresko, J. J., Jin, Z., Cheng, J., Huang, S., Sun, Y., Duschl, H., and Cheng, A. C. (2010). A wearable smartphone-based platform for real-time cardiovascular disease detection via electrocardiogram processing. IEEE Transactions on Information Technology in Biomedicine, 14(3):734-740.

Organization, W. H. et al. (2016). Hearts: technical package for cardiovascular disease management in primary health care.

Pedregosa, F., Varoquaux, G., Gramfort, A., Michel, V., Thirion, B., Grisel, O., Blondel, M., Prettenhofer, P., Weiss, R., Dubourg, V., Vanderplas, J., Passos, A., Cournapeau, D., Brucher, M., Perrot, M., and Duchesnay, E. (2011). Scikit-learn: Machine learning in Python. Journal of Machine Learning Research, 12:2825-2830.

Wong, T.-T. (2015). Performance evaluation of classification algorithms by k-fold and leave-one-out cross validation. Pattern Recognition, 48(9):2839-2846.

Xu, K., Guo, S., Cao, N., Gotz, D., Xu, A., Qu, H., Yao, Z., and Chen, Y. (2018). Ecglens: Interactive visual exploration of large scale ecg data for arrhythmia detection. In Proceedings of the 2018 CHI Conference on Human Factors in Computing Systems, page 663. ACM.

Zhao, R., Wang, D., Yan, R., Mao, K., Shen, F., and Wang, J. (2018). Machine health monitoring using local feature-based gated recurrent unit networks. IEEE Transactions on Industrial Electronics, 65(2):1539-1548. 